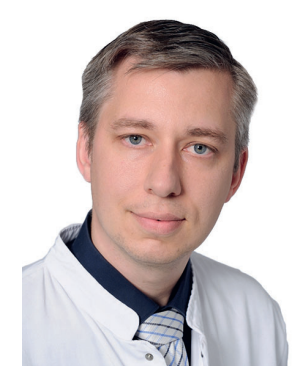

\title{
KAMRA-Inlays: Ursache von Trübungen sind Narbenreaktionen
}

Björn Bachmann

Zentrum für Augenheilkunde, Universität zu Köln, Köln, Deutschland

Abstract aus Paley GL, Harocopos GJ: Histopathologic analysis of explanted KAMRA corneal inlays demonstrating adherent fibroconnective tissue scar formation. Ocul Oncol Pathol 2019;DOI:10.1159/000498944

\section{Keywords}

Corneal inlay · Inlay explant · Fibroconnective tissue scar .

Presbyopia

\section{Abstract}

Objective: To investigate the histologic composition of opaque membranes associated with corneal intrastromal inlays implanted for the surgical treatment of presbyopia.

Methods: This is an observational case series of KAMRA corneal inlays explanted due to the presence of adherent opaque membranes associated with peri-inlay corneal stromal haze and sent for histopathologic analysis. Routine histology was performed in addition to immunohistochemical staining with myofibroblast and keratocyte markers.

Results: Eleven explanted inlay specimens were received, of which, after histologic processing, four demonstrated sufficient cellular material for histopathologic analysis. The opaque membranes surrounding the explanted inlays were composed of fibroconnective tissue, and myofibroblasts (positive for smooth muscle actin immunostain) were the predominant cell type. Immunostaining for the keratocyte marker CD34 was negative, confirming that the membranes were the result of a reactive scartissue formation process and not simply normal corneal stroma adherent to the explant.

Conclusions: Corneal inlay implantation can lead to the formation of an adherent fibroconnective tissue membrane, suggesting keratocyte-to-myofibroblast transdifferentiation and reactive fibroconnective tissue scar formation that could potentially impact visual potential. Prospective patients should be counseled regarding the risk of this complication, as this may be associated with some risk of incomplete reversibility of the procedure.

(c) 2019 S. Karger AG, Basel

\section{KARGER}

() 2019 S. Karger GmbH, Freiburg 


\section{Transfer in die Praxis}

\section{Hintergrund}

Die Presbyopie wird in Deutschland überwiegend mit einer Brille therapiert. Der Leidensdruck der Betroffenen kann sehr unterschiedlich, von gefühlt ohne Einschränkung bis zu sehr starker Störung, sein. Wenn die Presbyopie operativ korrigiert wird, geschieht dies häufig durch einen refraktiven Linsenaustausch bzw. im Rahmen der Kataraktoperation durch die Implantation multifokaler Intraokularlinsen. Einen alternativen Ansatz bei klarer Linse verfolgen intrastromale Hornhautimplantate, die nach Präparation eines Flaps oder einer Tasche im vorderen Hornhautstroma zentral platziert werden. Sie wirken z.B. durch Änderung der zentralen Refraktion der Hornhaut, wodurch ein Effekt ähnlich einer Multifokallinse entsteht. Das KAMRA-Inlay bedient sich dem Prinzip der stenopäischen Lücke, wodurch eine Verkleinerung der Apertur des Auges eine erhöhte Schärfentiefe erzielt. Es wird einseitig in die Hornhaut des typischerweise nicht dominanten Auge implantiert, sodass das Partnerauge weiterhin für die Entfernung genutzt werden kann. Ggf. muss der Eingriff noch mit einer Refraktionskorrektur im Sinne einer LASIK kombiniert werden. Durch die verkleinerte Apertur des Auges kann unter schlechten Lichtverhältnissen wie z.B. beim Dämmerungssehen die Sehleistung auch subjektiv störend reduziert sein. Als Vorteil der Implantate wird häufig die Reversibilität betont, da sich die Implantate zumindest in der ersten Zeit nach der Implantation häufig folgenlos entfernen lassen, sollte das refraktive Ergebnis bzw. der Seheindruck für den Patienten störend sein.

\section{Studienergebnisse}

Nach Implantation von intracornealen Implantaten kann es zu Trübungen um das Implantat kommen, die ebenfalls eine Explantation notwendig machen. In der vorliegenden Studie wurden 11
KAMRA-Inlays, die wegen Trübungen explantiert werden mussten, histologisch untersucht. Hierbei fanden die Autoren deutliche Hinweise darauf, dass die Trübungen auf eine Narbenreaktion um die Implantate herum zurückzuführen sind.

\section{Fazit für die Praxis}

Wie häufig Trübungen bzw. Narbenreaktionen nach intrastromalen Implantaten auftreten, ist nicht gut untersucht. In der Diskussion verweisen die Autoren auf eine FDA-Zulassungsstudie, die nach Implantation eine Rate von «klinisch signifikanten» Trübungen unter $1 \%$ innerhalb von 12 Monaten feststellte. Allerdings waren von dieser Analyse bereits 8,7\% der Patienten ausgenommen, bei denen das Implantat zu diesem Zeitpunkt bereits entfernt worden war. Den Anteil der Patienten, bei denen Trübungen/Vernarbungen auch jenseits des 12. Monats noch auftreten, ist ebenfalls nicht gut untersucht. Es gibt Berichte über Patienten, bei denen bis zu 6 Jahre nach Implantation Trübungen im Hornhautstroma eine Explantation notwendig machten. Auch nach Explantation verbleiben die Narben im anterioren Stroma und führen typischerwiese zu einer anhaltenden Sehverschlechterung, was bei der Patientenaufklärung berücksichtigt werden sollte.

\section{Disclosure Statement}

Kein Interessenkonflikt.

Kontaktadresse: Prof. Dr. Björn Bachmann, Zentrum für Augenheilkunde, Universitätsklinikum Köln, Kerpener Straße 62, 50937 Köln, Deutschland, bjoern.bachmann@uk-koeln.de 